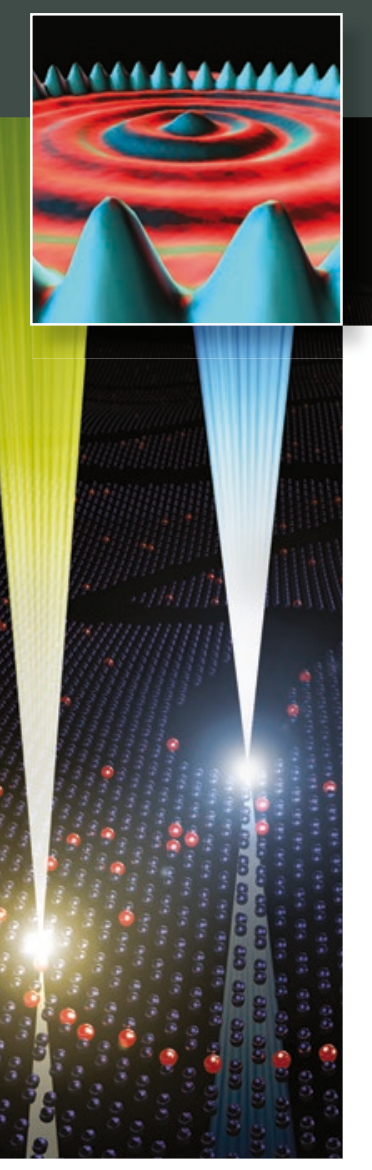

\title{
Single-atom fabrication with electron and ion beams: From surfaces and two- dimensional materials toward three- dimensional atom-by-atom assembly
}

\begin{abstract}
Sergei V. Kalinin and Stephen J. Pennycook, Guest Editors
The two current reigning paradigms enabling nanotechnology are scanning probe microscopy and molecular machine devices that date back to seminal experiments by Eigler and visionary work by Drexler, respectively. The nanoscience and nanotechnology community is seeing the emergence of a third paradigm - the use of the atomically focused beam of a scanning transmission electron microscope (STEM) to control and direct matter on the atomic scale. Beam-induced modifications involving one atom or a small group of atoms can be induced and monitored in real time with atomic resolution. Combined with the development of beam-control electronics, big data acquisition, and analytical tools such as artificial intelligence-based feedback systems, electron and ion microscopies are at the brink of a transition from purely imaging tools to tools capable of creating structures with atomic precision and high throughput. In this issue of MRS Bulletin, we present recent advances in electron- and ion-beam-based atomic fabrication on surfaces, in layered materials, and finally in three dimensions - the ultimate dream and possibly the final frontier of nanoscience.
\end{abstract}

\section{Introduction}

Ever since Democritus proposed the existence of atoms as the smallest indivisible unit of matter around $400 \mathrm{BC}$, this idea and its consequences have captured the attention of the scientific community. In the 19th century, broad adoption of atomistic theory led to remarkable progress in physics and chemistry, and eventually to the dawn of quantum physics in the early 20th century.

The earliest direct observation of atoms originally dates to the first field-ion microscope in the early 1950 s. $^{1}$ The first lattice fringes were observed in the 1950s by Menter using an electron microscope, building on the developments of Ruska, ${ }^{2}$ von Ardenne, ${ }^{3,4}$ and others, ${ }^{5}$ with gradually improving resolution over the following decades. Direct observation of individual atoms in scanning transmission electron microscopy (STEM) was achieved by Crewe and co-workers in the $1960 \mathrm{~s},{ }^{6}$ made possible by his development of the cold-fieldemission gun. ${ }^{7}$ Using an annular detector, direct imaging of atomic structures became possible, ${ }^{8-10}$ and in the last 30 years, the progress in STEM ${ }^{11}$ has rendered such observations routine. In comparison, scanning tunneling microscopy (STM) $)^{12-14}$ began with direct demonstration of atomic resolution on Si surfaces, even though earlier examples of current- and force-based mesoscopic profilometry are available.

Since the observation of atoms and the emergence of modern physics, a loftier dream has developed, as summarized by R. Feynman in his seminal talk "There is plenty of room at the bottom." ${ }^{15}$ The modern field of nanotechnology, however, owes much to the experimental work of D. Eigler at IBM ${ }^{16}$ and the visionary work of E. Drexler. ${ }^{17}$ The letters "I," "B," and "M" written in Xe atoms on a copper surface ${ }^{16}$ and the book "Engines of Creation"17 have provided the impetus by becoming firmly imprinted in societal perception of nanoscience as a pathway to control matter on the atomic scale, creating the machines and devices for ultimate medical devices and nanoassemblers. The evolution of social acceptance of nanotechnology is illustrated in Figure 1.

\section{The first paradigm: Molecular machines}

The first paradigm of nanotechnology was popularized by Drexler, who proposed the controlled chemical synthesis of 
molecular entities capable of autonomous motion and performing work, the nanomachines and nanoassemblers. ${ }^{17}$ This concept immediately captured the minds and imagination of broad swaths of the scientific community. ${ }^{18,19}$ Experimentally, efforts toward light, ${ }^{20,21}$ chemically ${ }^{22}$ and electrochemically ${ }^{23,24}$ activated molecular machines were undertaken, as recognized by the award of the 2016 Nobel Prize in Chemistry to J.-P. Sauvage, J. Fraser Stoddart, and B.L. Feringa.

Realization of this approach to nanoscience requires the simultaneous solution of three problems, namely, the design of molecular blocks with the required functionalities, the development of the synthetic pathways, and assembly in operational supramolecular structures. The power of modern computational methods makes the first viable, if not yet fully accomplished. Synthesis represents a more complex problem,

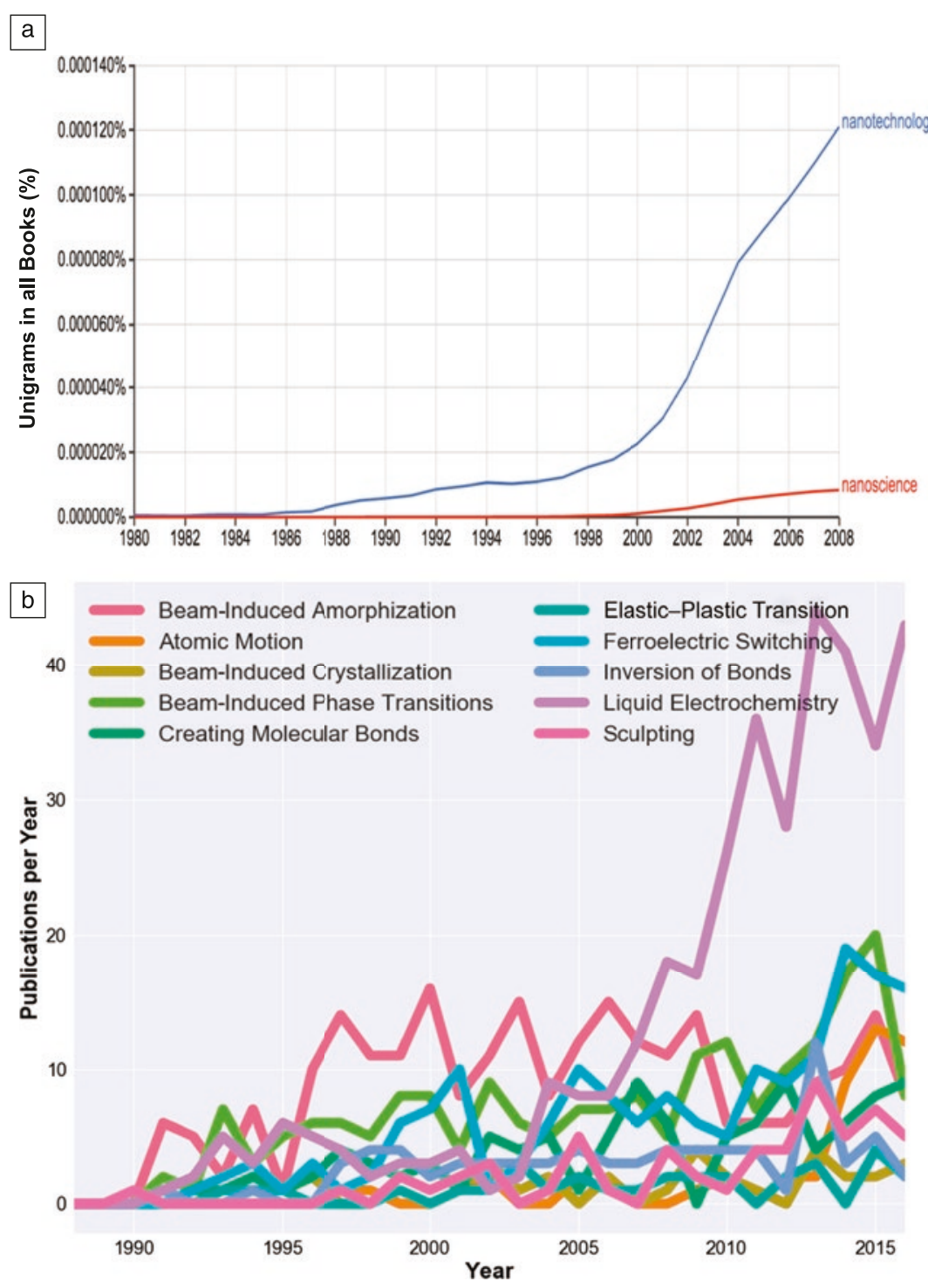

Figure 1. (a) Google Ngram search showing the growing frequency of the words "nanotechnology" and "nanoscience" in online books. (b) Number of publications per year on "electron microscopy" and (keyword), according to ISI. traditionally requiring outstanding organic synthesis intuition and broad knowledge base. However, the recent establishment of reaction databases combined with advances in graphical search algorithms has enabled automatic identification of synthetic pathways for all known and many unknown (e.g., via retrosynthesis) compounds. ${ }^{25,26}$ However, it is the probing and assembly of molecular machines into operational structures that remains a central issue in the field.

\section{The second paradigm: Scanning probe manipulation}

The second paradigm of nanotechnology is based on direct atomic manipulation by scanning probes. From the early days of scanning tunneling microscopy, it was realized that the probe tip can induce the motion of loosely bound atoms on material surfaces. In some cases, the surface can be imaged prior to and after the manipulation, providing atomically resolved views of surface changes. This provided the ultimate opportunity to study cause and effect on the atomic scale.

The real breakthrough came with the experiments of Eigler at IBM, who developed an approach to position deposited atoms in predefined configurations. ${ }^{16}$ In the 25 years since this work, the field has seen multiple advances in fabrication, basic physics, and societal impact. Some of the breakthrough concepts introduced by the Eigler group include quantum corrals ${ }^{27}$ and molecular cascades,${ }^{28}$ opening pathways for probing the fundamental physics of quantum states in real space and molecular motion-based computational devices. Other advances, including ultrahigh density storage $^{29}$ and holographic memories have been reported. The movie The Boy and His Atom ${ }^{30}$ captured public interest in this, collecting more than $6 \mathrm{M}$ views on YouTube. Many of these advances are summarized in a number of recent reviews, and some of the highlights are shown in Figure 2. ${ }^{31-35}$

The fundamental limitation of STM-based fabrication is that in many cases, it is limited to low temperatures, with liquid helium temperature $(4 \mathrm{~K})$ being the norm, and requiring atomically flat clean surfaces. These approaches are also fairly slow and have limited throughput. At the same time, mainstream nanotechnology necessitates room-temperature stability (either at operational temperatures or intermediate fabrication steps), and reasonably fast fabrication. Simmons et al. ${ }^{36,37}$ and several other groups demonstrated the achievement of this goal by a combination of STM manipulation with classical surface science techniques for fabrication of atomically defined surfaces. 


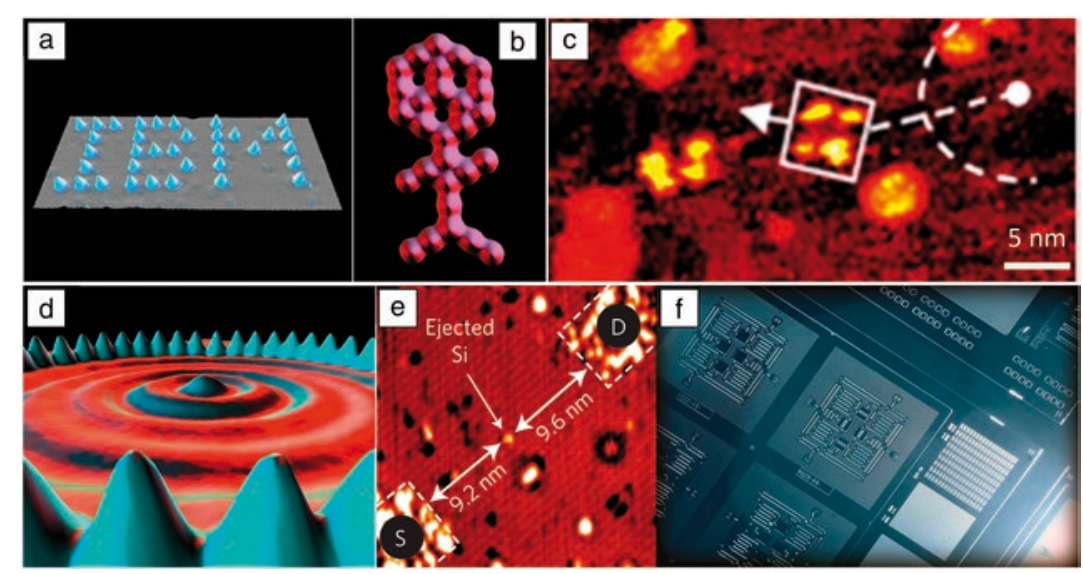

Figure 2. Examples of single-atom manipulation with scanning probes. (a) Xenon atoms on nickel(110) surface forming "IBM." Image originally created by IBM Corporation. (b) Carbon monoxide man on platinum(111). Image originally created by IBM Corporation. (c) Scanning tunneling micrograph of a four-wheeled "nanocar" on a Au(111) surface. Adapted with permission from Reference 33. (c) 2005 American Chemical Society. (d) Quantum corral, iron on copper(111). Image originally created by IBM Corporation. (e) Single-atom transistor with a single Si atom. Note: S, source; D, drain. Reprinted with permission from Reference 34. (C) 2012 Nature Publishing Group. (f) Nb and Al circuits on a Si substrate, forming a multiple-qubit device. Reprinted with permission from Reference 35. (c) 2015 Nature Publishing Group.

\section{The third paradigm: Electron beams}

The introduction of high-resolution aberration-corrected electron microscopy and related progress in electron energy-loss spectroscopy (EELS) in the early 2000s have revolutionized the field of condensed-matter physics and materials science. Following the initial demonstration of single-atom sensitivity in EELS $^{38}$ and three-dimensional (3D) imaging capability via focal series, ${ }^{39}$ the increased spatial resolution and sensitivity of STEM have enabled advances such as direct mapping of polarization, ${ }^{40,41}$ in-plane octahedral tilts, ${ }^{42}$ and chemical strains, ${ }^{43,44}$ and was recently extended to probe tilt systems in the $z$-direction. ${ }^{45}$ Advances in the quantification of STEM have determined the location of single vacancy centers, ${ }^{46}$ and are likely to lead to further breakthroughs. In parallel with

\begin{tabular}{|l|c|}
\hline \multicolumn{1}{|c|}{ Table I. Atomistic and nanoscale beam-induced phenomena. } \\
\hline \multicolumn{1}{|c|}{ Phenomon } & Reference \\
\hline Crystallization of amorphous material & $59-61$ \\
\hline Elastic-plastic transition & 49 \\
\hline Ferroelectric domain switching & 62 \\
\hline Phase transitions & 63,64 \\
\hline Vacancy formation and dynamics & 65 \\
\hline Creating of molecular bonds & 66 \\
\hline Inversion of bonds & 67 \\
\hline Atomic motion & 68 \\
\hline Sculpting (erosion) & 55 \\
\hline Liquid electrochemistry & 69 \\
\hline
\end{tabular}

the mainstream development of STEM as a purely imaging/spectroscopy tool, a number of groups have explored the potential of atomically focused beams for resist-based lithography, ${ }^{47,48}$ now approaching 1-nm resolution, and e-beam deposition. However, in the vast majority of work to date, STEM has been perceived only as an imaging tool, and any beam-induced modifications were viewed as undesirable beam damage.

A brief historical overview of e-beam, ion beam, and particle beam literature suggests that during the last three decades, these beams were found to induce significant modification in the structure of solids. One example is beaminduced crystallization and amorphization. This area was actively explored in the 1980s and 1990s, and e-beam crystallization of a number of important semiconductors such as $\mathrm{Si}^{49-52}$ and $\mathrm{GaAs}^{52-54}$ has been reported. Similarly, the beam can result in selective removal of material, and when integrated with beam-induced reactions, it can enable fabrication of nanoscale structures, as summarized in recent reviews by Krasheninnikov, ${ }^{55}$ Gonzales-Martinez, ${ }^{56}$ and Jesse. ${ }^{57}$ The associated mechanisms are discussed by Jiang. ${ }^{58}$ However, these beam-fabrication processes primarily explored mesoscopic-level changes in materials structure, as limited by the electron-microscopy platforms of the time.

In the last five years, the proliferation of high-resolution STEMs and their intrinsic propensity for beam-induced modifications in solids have led several groups to explore and report atomic-level beam-induced modifications, including phase transitions, vacancy creation, and atomic motion, as summarized in Table I. ${ }^{49,55,59-69}$ Some of these are further illustrated in Figure 3. ${ }^{62,70-76}$ What is remarkable is that these changes often involve one atom or small groups of atoms, and can be monitored in real time with atomic resolution. Further examples of controllable electron-beam-induced phenomena are discussed throughout this issue.

This broad range of well-defined beam-induced processes suggests tremendous potential for materials science, chemistry, and nanofabrication. Some examples of direct e-beam fabrication are illustrated in Figure 4. ${ }^{77-79}$ Imagine being able to implement the same spectrum of phenomena - or more-with an electron beam in the bulk as with scanning probe microscopy on a material's surface - from fabrication of single-atom devices, atom-by-atom assembly, editing defect configurations in solids, to the realization of electron-beam-driven molecular motors, and more.

At this point, we are only at the beginning of this pathway. The observed phenomena at this point are poorly controlled (e.g., both crystallization and amorphization can be induced by electron beams for slightly different parameters, but we don't know why or how to predict it), and the associated mechanisms acting at the atomic level are poorly understood. 

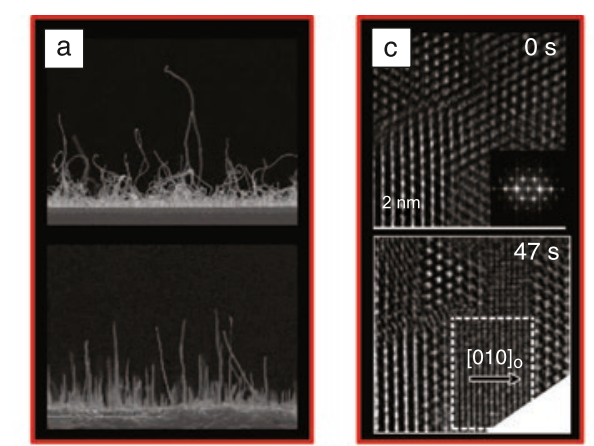

Phase

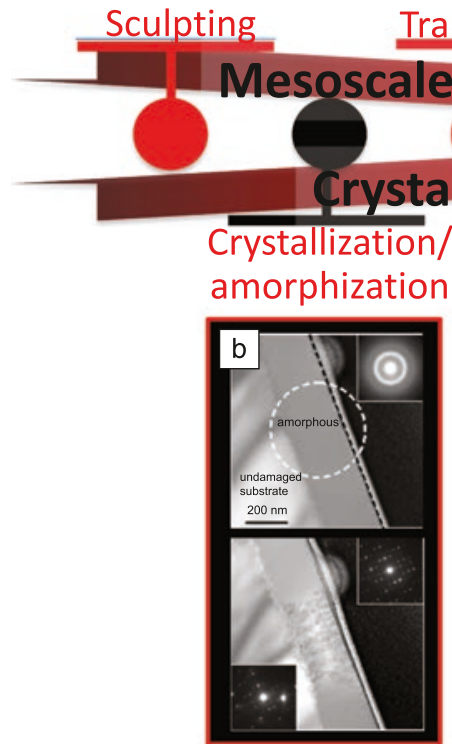

Figure 3. Examples of the electron-beam- and ion-beam-induced changes in materials structure resolved at atomic or near-atomic level. (a) Sculpting: scanning electron microscope images before and after straightening carbon nanotubes by Ar ion irradiation. Reprinted with permission from Reference 70. (c) 2003 Elsevier. (b) Crystallization/amorphization: structural changes of $\mathrm{Sr}_{2} \mathrm{Nd}_{8}\left(\mathrm{SiO}_{4}\right)_{6} \mathrm{O}_{2}$ under electron irradiation. Reprinted with permission from Reference 60. (C) 2007 AIP Publishing. (c) Phase transitions: phase transition from $\gamma$-CaSo ${ }_{4}$ to $\beta-\mathrm{CaSO}_{4}$. Adapted with permission from Reference 71. (C) 2015 American Chemical Society. (d) Atomic column rearrangement: annular darkfield images showing phase front advancement in transition from $\mathrm{Mn}_{3} \mathrm{O}_{4}$ to $\mathrm{MnO}^{72}$ (e) Domain switching: electron-beam-induced domain switching in Rb-doped $\mathrm{KTiOPO}_{4}$. Reprinted with permission from Reference 62. (c) 2016 American Physical Society. (f) Bond formation: experimental (right) and simulated (left) images of bond formation in perchlorocoronene under electron-beam irradiation. Adapted with permission from Reference 73. (C) 2017 American Chemical Society. (g) Vacancy formation in graphene sheet after irradiation by a focused

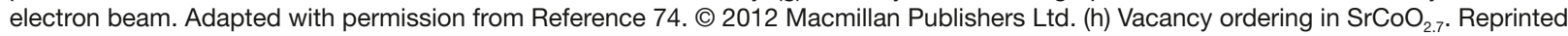
with permission from Reference 75 . (i) Atomic motion: movement of Ce dopant laterally within wurtzite-type aluminum nitride. ${ }^{76}$

Achieving further progress and harnessing beam-induced atomic motion and fabrication for nanofabrication requires solving a set of interlinked problems. The first is the generation of forward-predictive models for electron-beam-matter interactions that take into account both elastic and inelastic scattering events and allow calculation of the sequence of changes in electronic and atomic structure of materials during electron irradiation. Such models are intrinsically complex since they aim to link the stochastic aspects of energy transfer from $\mathrm{keV}$ electrons with the electronic and ionic subsystem over a time scale ranging from femtoseconds to seconds. Development of two temperature or equivalent models coupled with molecular dynamics simulations is a step in the right direction. The key step forward from the present state of the art will be to transition from single-particle simulations to the effects of multiple particles in a larger volume that will allow researchers to elucidate the statistical parameters of the process, classify the probabilities of atomic knock-on events and resulting displacements, and also quantify low-probability processes. Similarly of interest are the models that explore the evolution of charges and electron and ion currents in the irradiated area.

Complementary to this will be the creation of a library of structures and beam-induced transformations based on experimental observations, akin to the reaction pathway analysis in biochemical and catalysis communities. This will, in turn, necessitate fully harnessing data flow from detectors, the creation of rapid image analytic tools to identify observed atomic 

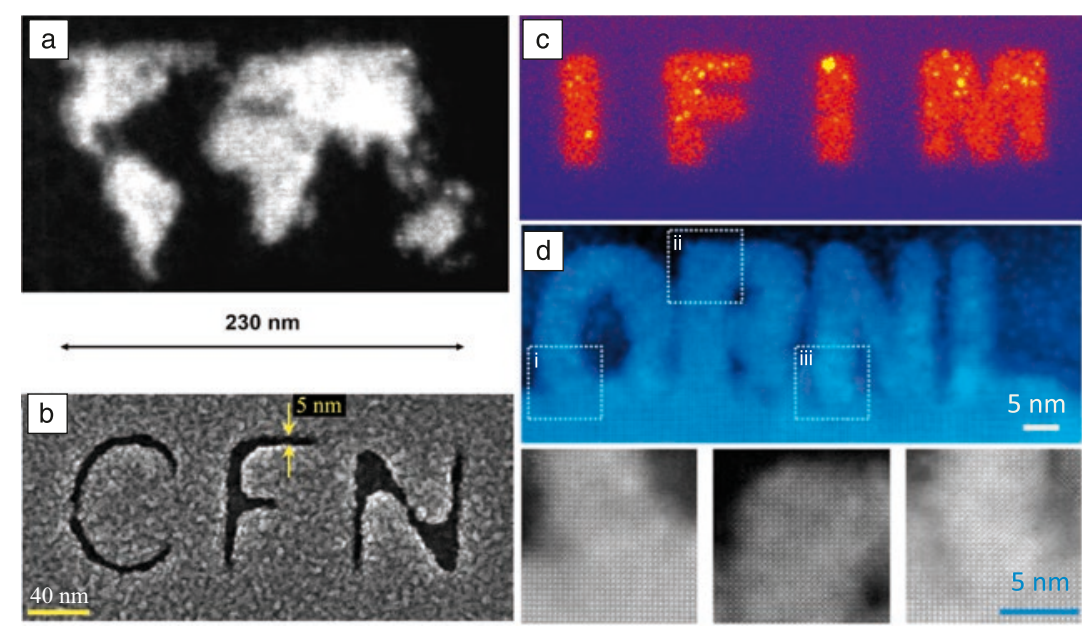

Figure 4. Examples of electron-beam-based fabrication. (a) Scanning transmission electron microscope image of a nanoscale topographic map of the world drawn using electronbeam-induced deposition and a tungsten precursor. Adapted with permission from Reference 77. (c) 2008 World Scientific. (b) Aberration-corrected electron-beam lithography in poly(methyl methacrylate). ${ }^{78}$ (c) IFIM. (d) Text "ORNL" patterned on the interface of amorphous and crystalline $\mathrm{SrTiO}_{3}{ }^{79}$

to quantum and exotic phonon devices. While the throughput of the e-beam (as any sequential) technique will be limited, it is likely to surpass STM-based fabrication in terms of speed. Further introduction of multibeam systems can accelerate the enabling process (e.g., 100-qubit device fabrication). Initial progress is likely to be assisted by a large fleet of extant STEM platforms that can be repurposed for these applications.

\section{In this Issue}

This issue of MRS Bulletin assembles contributions that detail recent progress in electron- and ion-beam fabrication on the atomic level. In their article in this issue, Mishra et al. ${ }^{83}$ provide an overview of the energy and momentum transfer processes in STEM, and further summarize recent observations of electronbeam-induced single-atom dynamics in STEM. In their article, Zhao et al. ${ }^{84}$ report on e-beaminduced modifications in the atomic structure of two-dimensional materials such as graph-

structures from local imaging, also ptychography (imaging based on analyzing the diffraction signal from STEM), ${ }^{80-82}$ and establishing common knowledge spaces to integrate information from multiple microscopic platforms (similar to research models in astronomy or genomics). This requires deconvolution of the microscope transfer function (a measure of resolution and performance) and calibration, so that materialsspecific phenomena are separated from instrumental factors. It also requires establishing common file formats and analysis tools. When available, these experimental cause and effect libraries can be compared and used to improve theoretical ones. With the information on cause and effect at hand, advances in machine learning can be used to produce required beam trajectories and parameters to enable atomic-level fabrication.

\section{Perspective}

Electron-beam control and direction of matter will yield multiple research opportunities in areas spanning basic and applied science and nanofabrication. In basic science research, the dual potential of STEMs to image atomic structure and probe associated electronic properties combined with beam atom-by-atom fabrication opens pathways to create new atomic configurations and probe their electronic, plasmonic, and phononic properties. Similarly, while the early stages of e-beaminduced phenomena are intrinsic to this field, observations of later stages of molecular, vacancy, and single-atom dynamics can provide insights into atomically resolved mechanisms of phase transitions, chemical, and electrochemical reactions.

Similarly, the ability to create predefined atomic configurations in the bulk, or edit STM- or lithographically fabricated devices offers tremendous potential for atomically precise device fabrication, ranging from single-spin magnetoelectronics ene and layered chalcogenides, including creation of single vacancies and defect clusters, beam-induced phase transitions, and single-atom motion. In their article, Belianinov et al. ${ }^{85}$ report on ion-beam-based matter fabrication, advances enabled by rapidly emerging $\mathrm{He}$ and other ion microscopies. Finally, Jiang et al. ${ }^{86}$ report on the applications of electron beams in inducing local structural changes in 3D solids, including amorphization-crystallization and phase transitions. Combined with beam control, this enables fabrication of $3 \mathrm{D}$ atomic structures with single-atomic plane precision.

\section{Summary}

Feynman famously said, "There's plenty of room at the bottom." 15 Modern microelectronics development has allowed scientists to "think" at the "bottom," or the nanoscale, as exemplified by sub-10-nm semiconductor and emerging quantum-computing technologies. ${ }^{37}$ However, the realization of the vision of nanoscience-from nanorobotics to destroy cancer cells to reconfigurable electronics - also requires the capability to "act" and "build" on the nanoscale. The two primary paradigms of nanotechnology-scanning probe-based fabrication and chemical synthesis and self-assembly-have been guiding the development of this field for more than two decades. Now, a third paradigm, the "atom forge" 81,82 — a toolbelt of electron-beam-based methods for direct atomic manipulation and atom-by-atom assembly - joins this field.

\section{Acknowledgments}

This research was conducted and partially supported (S.V.K.) at the Center for Nanophase Materials Sciences, which is a US Department of Energy Office of Science User Facility. The authors gratefully acknowledge assistance from A. Maksov 
(The University of Tennessee, Knoxville) with figure preparation and literature analysis. S.J.P. is grateful to the National University of Singapore for support.

\section{References}

1. E.W. Muller, J. Appl. Phys. 27 (5), 474 (1956)

2. M. Knoll, E. Ruska, Z. Phys. A Hadrons Nucl. 78, 318 (1932).

3. M. von Ardenne, Z. Phys. 109 (9-10), 553 (1938).

4. M. von Ardenne, Z. Tech. Phys. 19, 407 (1938).

5. J. Menter, Proc. R. Soc. Lond. A 236 (1204), 119 (1956).

6. A.V. Crewe, J. Wall, J. Langmore, Science 168 (3937), 1338 (1970).

7. A.V. Crewe, D.N. Eggenberger, J. Wall, L.M. Welter, Rev. Sci. Instrum. 39 (4), 576 (1968).

8. J. Wall, J. Langmore, M. Isaacson, A.V. Crewe, Proc. Natl. Acad. Sci. U.S.A. 71 (1), 1 (1974)

9. S.J. Pennycook, L.A. Boatner, Nature 336 (6199), 565 (1988).

10. S.J. Pennycook, D.E. Jesson, Phys. Rev. Lett. 64 (8), 938 (1990).

11. S.J. Pennycook, M. Varela, J. Electron Microsc. 60, S213 (2011)

12. G. Binnig, C.F. Quate, C. Gerber, Phys. Rev. Lett. $\mathbf{5 6}$ (9), 930 (1986)

13. G. Binnig, H. Rohrer, C. Gerber, E. Weibel, Phys. Rev. Lett. 50 (2), 120 (1983).

14. G. Binnig, H. Rohrer, Helv. Phys. Acta 55 (6), 726 (1982).

15. R.P. Feynman, Caltech Eng. Sci. 23 (5), 22 (1960).

16. D.M. Eigler, E.K. Schweizer, Nature 344 (6266), 524 (1990)

17. E. Drexler, Engines of Creation: The Coming Era of Nanotechnology (Anchor, New York, 1987)

18. A. Coskun, M. Banaszak, R.D. Astumian, J.F. Stoddart, B.A. Grzybowski, Chem. Soc. Rev. 41 (1), 19 (2012).

19. W.R. Browne, B.L. Feringa, Nat. Nanotechnol. 1 (1), 25 (2006).

20. N. Ruangsupapichat, N.M. Pollard, S.R. Harutyunyan, B.L. Feringa, Nat Chem. 3 (1), 53 (2011).

21. S. Saha, J.F. Stoddart, Chem. Soc. Rev. 36 (1), 77 (2007).

22. S.P. Fletcher, F. Dumur, M.M. Pollard, B.L. Feringa, Science 310 (5745), 80 (2005)

23. V. Balzani, M. Gomez-Lopez, J.F. Stoddart, Acc. Chem. Res. 31 (7), 405 (1998).

24. T. Kudernac, N. Ruangsupapichat, M. Parschau, B. Maciá, N. Katsonis S.R. Harutyunyan, K.H. Ernst, B.L. Feringa, Nature 479 (7372), 208 (2011).

25. S. Szymkuć, E.P. Gajewska, T. Klucznik, K. Molga, P. Dittwald, M. Startek, M. Bajczyk, B.A. Grzybowski, Angew. Chem. Int. Ed. 55 (20), 5904 (2016).

26. A. Cadeddu, E.K. Wylie, J. Jurczak, M. Wampler-Doty, B.A. Grzybowski, Angew. Chem. Int. Ed. $\mathbf{5 3}$ (31), 8108 (2014).

27. E.J. Heller, M.F. Crommie, C.P. Lutz, D.M. Eigler, Nature 369 (6480), 464 (1994)

28. A.J. Heinrich, C.P. Lutz, J.A. Gupta, D.M. Eigler, Science 298 (5597), 1381 (2002)

29. F. Donati, S. Rusponi, S. Stepanow, C. Wäckerlin, A. Singha, L. Persichetti, R. Baltic, K. Diller, F. Patthey, E. Fernandes, J. Dreiser, Science 352 (6283), 318 (2016).

30. IBM, "A Boy and His Atom: The World's Smallest Movie," YouTube video, 1:33, posted April 30, 2013, https://www.youtube.com/watch?v=0SCX78-8-q0.

31. K. Morgenstern, N. Lorente, K.H. Rieder, Phys. Status Solidi B 250 (9), 1671 (2013).

32. S.W. Hla, Rep. Prog. Phys. 77 (5), (2014)

33. Y. Shirai, A.J. Osgood, Y. Zhao, K.F. Kelly, J.M. Tour, Nano Lett. 5 (11), 2330 (2005)

34. M. Fuechsle, J.A. Miwa, S. Mahapatra, H. Ryu, S. Lee, O. Warschkow, L.C. Hollenberg, G. Klimeck, M.Y. Simmons, Nat. Nanotechnol. 7 (4), 242 (2012).

35. S. Benjamin, J. Kelly, Nat. Mater. 14 (6), 561 (2015).

36. S.R. Schofield, N.J. Curson, M.Y. Simmons, F.J. Rueß, T. Hallam, L. Oberbeck, R.G. Clark, Phys. Rev. Lett. 91 (13), 136104 (2003).

37. M. Fuechsle, J.A. Miwa, S. Mahapatra, H. Ryu, S. Lee, O. Warschkow, L.C. Hollenberg, G. Klimeck, M.Y. Simmons, Nat. Nanotechnol. 7 (4), 242 (2012).

38. M. Varela, S.D. Findlay, A.R. Lupini, H.M. Christen, A.Y. Borisevich, N. Dellby, O.L. Krivanek, P.D. Nellist, M.P. Oxley, L.J. Allen, S.J. Pennycook, Phys. Rev. Lett. 92 (9), 095502 (2004).

39. A.Y. Borisevich, A.R. Lupini, S.J. Pennycook, Proc. Natl. Acad. Sci. U.S.A. 103 (9), 3044 (2006)

40. H.J. Chang, S.V. Kalinin, A.N. Morozovska, M. Huijben, Y.H. Chu, P. Yu, R. Ramesh, E.A. Eliseev, G.S. Svechnikov, S.J. Pennycook, A.Y Borisevich, Adv. Mater. 23 (21), 2474 (2011).

41. J. Chun-Lin, V. Nagarajan, H. Jia-Qing, L. Houben, T. Zhao, R. Ramesh, K. Urban, R. Waser, Nat. Mater. 6 (1), 64 (2007).

42. A.Y. Borisevich, H.J. Chang, M. Huijben, M.P. Oxley, S. Okamoto, M.K. Niranjan, J.D. Burton, E.Y. Tsymbal, Y.H. Chu, P. Yu, R. Ramesh, Phys. Rev. Lett. 105 (8), (2010).
43. Y.M. Kim, J. He, M.D. Biegalski, H. Ambaye, V. Lauter, H.M. Christen, S.T. Pantelides, S.J. Pennycook, S.V. Kalinin, A.Y. Borisevich, Nat. Mater. 11 (10), 888 (2012).

44. A.B. Yankovich, B. Berkels, W. Dahmen, P. Binev, S.I. Sanchez, S.A. Bradley, A. Li, I. Szlufarska, P.M. Voyles, Nat. Commun. 5, 4155 (2014)

45. Q. He, R. Ishikawa, A.R. Lupini, L. Qiao, E.J. Moon, O. Ovchinnikov, S.J. May, M.D. Biegalski, A.Y. Borisevich, ACS Nano 9 (8), 8412 (2015).

46. D.Y. Kim, S.Z. Lin, F. Weickert, M. Kenzelmann, E.D. Bauer, F. Ronning, J.D. Thompson, R. Movshovich, Phys. Rev. X6 (4), 041059 (2016).

47. V.R. Manfrinato, J. Wen, L. Zhang, Y. Yang, R.G. Hobbs, B. Baker, D. Su, D. Zakharov, N.J. Zaluzec, D.J. Miller, E.A. Stach, Nano Lett. 14 (8), 4406 (2014) 48. V.R. Manfrinato, L. Zhang, D. Su, H. Duan, R.G. Hobbs, E.A. Stach, K.K Berggren, Nano Lett. 13 (4), 1555 (2013).

49. S. Dai, J. Zhao, L. Xie, Y. Cai, N. Wang, J. Zhu, Nano Lett. 12 (5), 2379 (2012) 50. D. Amkreutz, J. Müller, M. Schmidt, T. Hänel, T.F. Schulze, Prog. Photovolt. 19 (8), 937 (2011)

51. Z.L. Wang, N. Itoh, N. Matsunami, Q.T. Zhao, Nucl. Instrum. Methods Phys. Res. B100 (4), 493 (1995)

52. I. Jencic, M.W. Bench, I.M. Robertson, M.A. Kirk, J. Appl. Phys. 78 (2), 974 (1995).

53. X. Yang, R. Wang, H. Yan, Z. Zhang, Mater. Sci. Eng. B 49 (1), 5 (1997)

54. Z.C. Li, Acta Metall. Sinica 39 (1), 13 (2003)

55. A.V. Krasheninnikov, K. Nordlund, J. Appl. Phys. 107 (7), 3 (2010)

56. I.G. Gonzalez-Martinez, A. Bachmatiuk, V. Bezugly, J. Kunstmann, T. Gemming, Z. Liu, G. Cuniberti, M.H. Rümmeli, Nanoscale 8 (22), 11340 (2016).

57. S. Jesse, A.Y. Borisevich, J.D. Fowlkes, A.R. Lupini, P.D. Rack, R.R. Unocic B.G. Sumpter, S.V. Kalinin, A. Belianinov, O.S. Ovchinnikova, ACS Nano 10 (6), 5600 (2016)

58. N. Jiang, Rep. Prog. Phys. 79 (1), (2016).

59. S. Jesse, Q. He, A.R. Lupinin, D. Leonard, M.P. Oxley, O. Ovchinnikov, R.R. Unocic, A. Tselev, M. Fuentes-Cabrera, B.G. Sumpter, S.J. Pennycook, S.V. Kalinin, A.Y. Borisevich, Small 11 (44), 5895 (2015).

60. I.T. Bae, Y. Zhang, W.J. Weber, M. Higuchi, L.A. Giannuzzi, Appl. Phys. Lett. 90 (2), 021912 (2007)

61. Y. Zhang, J. Lian, C.M. Wang, W. Jiang, R.C. Ewing, W.J. Weber, Phys. Rev $B$ Condens. Matter 72 (9), 094112 (2005).

62. J.L. Hart, S. Liu, A.C. Lang, A. Hubert, A. Zukauskas, C. Canalias, R. Beanland, A.M. Rappe, M. Arredondo, M.L. Taheri, Phys. Rev. B Condens. Matter 94 (17), 174104 (2016).

63. F. Cao, H. Zheng, S. Jia, X. Bai, L. Li, H. Sheng, S. Wu, W. Han, M. Li, G. Wen J. Yu, J. Phys. Chem. C119 (38), 22244 (2015).

64. H. Zheng, J.B. Rivest, T.A. Miller, B. Sadtler, A. Lindenberg, M.F. Toney, L.W. Wang, C. Kisielowski, A.P Alivisatos, Science 333 (6039), 206 (2011)

65. H.-P. Komsa, J. Kotakoski, S. Kurasch, 0. Lehtinen, U. Kaiser, A.V. Krasheninnikov, Phys. Rev. Lett. 109 (3), 035503 (2012).

66. Y. Ding, Y. Chen, K.C. Pradel, M. Liu, Z.L. Wang, J. Appl. Phys. 120 (21), $214302(2016)$

67. Z. Yang, L. Yin, J. Lee, W. Ren, H.M. Cheng, H. Ye, S.T. Pantelides, S.J. Pennycook, M.F. Chisholm, Angew. Chem. Int. Ed. 53 (34), 8908 (2014). 68. R. Ishikawa, R. Mishra, A.R. Lupini, S.D. Findlay, T. Taniguchi, S.T. Pantelides, S.J. Pennycook, Phys. Rev. Lett. 113 (15), 155501 (2014).

69. R.R. Unocic, A.R. Lupini, A.Y. Borisevich, D.A. Cullen, S.V. Kalinin, S. Jesse, Nanoscale 8 (34), 15581 (2016).

70. D.H. Kim, H.S. Jang, C.D. Kim, D.S. Cho, H.D. Kang, H.R. Lee, Chem. Phys. Lett. 378 (3-4), 232 (2003).

71. F. Cao, H. Zheng, S. Jia, X. Bai, L. Li, H. Sheng, S. Wu, W. Han, M. Li, G. Wen, J. Yu, J. Phys. Chem. C 119 (38), 22244 (2015)

72. T.J. Pennycook, L. Jones, H. Pettersson, J. Coelho, M. Canavan, B. MendozaSanchez, V. Nicolosi, P.D. Nellist, Sci. Rep. 4, 7555 (2014).

73. T.W. Chamberlain, J. Biskupek, S.T. Skowron, A.V. Markevich, S. Kurasch, 0. Reimer, K.E. Walker, G.A. Rance, X. Feng, K. Müllen, A. Turchanin, M.A. Lebedeva A.G. Majouga, V.G. Nenajdenko, U. Kaiser, E. Besley, A.N. Khlobystov, ACS Nano 11 (3), 2509 (2017).

74. A.W. Robertson, C.S. Allen, Y.A. Wu, K. He, J. Olivier, J. Neethling, A.I. Kirkland, J.H. Warner, Nat. Commun. 3, 1144 (2012).

75. J.T. Mefford, X. Rong, A.M. Abakumov, W.G. Hardin, S. Dai, A.M. Kolpak, K.P. Johnston, K.J. Stevenson, Nat. Commun. 7, 11053 (2016).

76. R. Ishikawa, A.R. Lupini, S.D. Findlay, T. Taniguchi, S.J. Pennycook, Nano Lett. 14 (4), 1903 (2014)

77. V.R. Manfrinato, A. Stein, L. Zhang, C.Y. Nam, K.G. Yager, E.A. Stach, C.T. Black, Nano Lett. (forthcoming).

78. S.V. Kalinin, A. Borisevich, S. Jesse, Nature 539 (7630), 485 (2016).

79. S.V. Kalinin, Foresight Institute, "Atomic Forge-Foresight Institute." YouTube video, 5:25., posted February 14, 2017, https://www.youtube.com/ watch?v=mZMhRPAJRsw.

80. J.M. Rodenburg, B.C. McCallum, P.D. Nellist, Ultramicroscopy 48 (3), 304 (1993). 
81. P.D. Nellist, B.C. McCallum, J.M. Rodenburg, Nature 374, 630 (1995).

82. F. Hüe, J.M. Rodenburg, A.M. Maiden, F. Sweeney, P.A. Midgley, Phys. Rev. B 82, 121415, (2010).

83. R. Mishra, R. Ishikawa, A.R. Lupini, S.J. Pennycook, MRS Bull. 42 (9), 644 (2017).

84. X. Zhao, J. Kotakoski, J.C. Meyer, E. Sutter, P. Sutter, A.V. Krasheninnikov, U. Kaiser, W. Zhou, MRS Bull. 42 (9), 667 (2017).

85. A. Belianinov, M.J. Burch, S. Kim, S. Tan, G. Hlawacek, O.S. Ovchinnikova, MRS Bull. 42 (9), 660 (2017).

86. N. Jiang, E. Zarkadoula, P. Narang, A. Maksov, I. Kravchenko, A. Borisevich, S. Jesse, S.V. Kalinin, MRS Bull. 42 (9), 653 (2017).

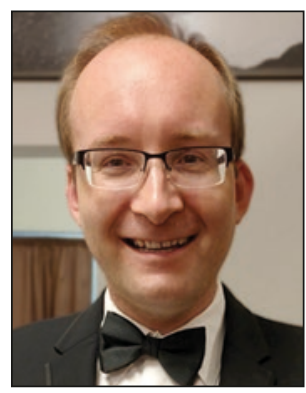

Sergei V. Kalinin is the director of the Oak Ridge National Laboratory (ORNL) Institute for Functional Imaging of Materials and a distinguished research staff member at the Center for Nanophase Materials Sciences at ORNL, since 2002. He also holds a joint associate professor position in the Department of Materials Science and Engineering at The University of Tennessee, Knoxville. He received his $\mathrm{PhD}$ degree from the University of Pennsylvania in 2002, followed by a Wigner fellowship at ORNL (2002-2004). His research interests include application of big data, deep data, and smart data approaches in atomically resolved and mesoscopic imaging to guide the development of advanced materials for energy and information technologies, as well as electromechanical, electrical, and transport phenomena and matter patterning on the nanoscale via scanning probe and electron microscopy. He is a recipient of several awards, including the RMS Medal for Scanning Probe Microscopy (2015) and the Presidential Early Career Award for Scientists and Engineers (2009); three R\&D 100 Awards (2008, 2010, and 2016); and a number of other distinctions. He has published more than 500 papers, edited three books, and holds more than 16 patents. Kalinin can be reached by email at sergei2@ornl.gov.

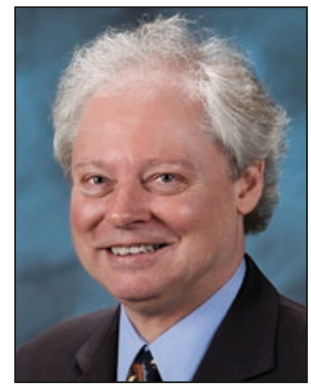

Stephen J. Pennycook is a professor in the Department of Materials Science and Engineering, National University of Singapore, an adjunct professor at The University of Tennessee, and adjoint professor at Vanderbilt University. Previously, he was Corporate Fellow in the Materials Science and Technology Division of Oak Ridge National Laboratory and leader of the Scanning Transmission Electron Microscopy Group. He completed his PhD degree in physics at the Cavendish Laboratory, University of Cambridge, UK, in 1978. Since then, he has been actively pursuing the development and materials applications of atomic resolution Z-contrast microscopy and electron energy-loss spectroscopy. Pennycook is a Fellow of the Materials Research Society, the American Physical Society, the American Association for the Advancement of Science, the Microscopy Society of America, and the Institute of Physics. He has received the Microbeam Analysis Society Heinrich Award, the Materials Research Society Medal, the Institute of Physics Thomas J. Young Medal and Award, and the Materials Research Society Innovation in Materials Characterization Award. He has published 38 books and book chapters, and more than 500 papers and 250 invited presentations. His latest book is Scanning Transmission Electron Microscopy. Pennycook can be reached by phone at $+(65) 6516-5193$ or by email at steve.pennycook@nus.edu.sg or msepsj@nus.edu.sg.

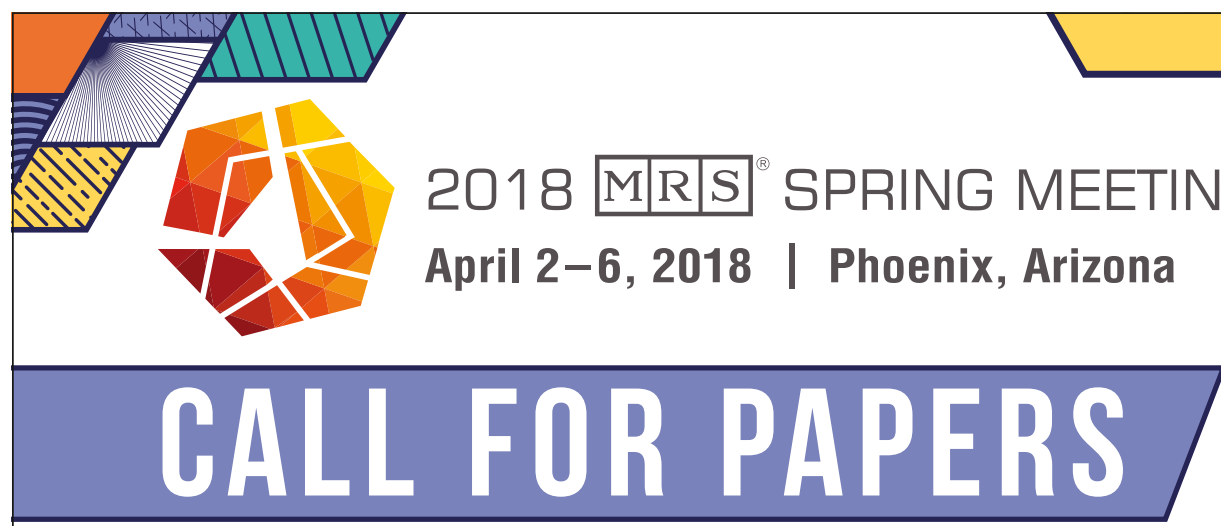

Abstract Submission Opens September 29, 2017

Abstract Submission Deadline October 31, 2017

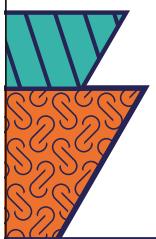

The program features 61 topical symposia in the following clusters:

- Characterization, Modeling and Theory

- Electronic and Photonic Materials

- Energy Materials and Technologies

- Manufacturing

- Nanomaterials

- Soft Materials and Biomaterials

Meeting Chairs

Edward Botchwey Georgia Institute of Technology/Emory University

Catherine Dubourdieu Helmholtz-Zentrum Berlin

Quanxi Jia University at Buffalo, The State University of

New York/Los Alamos National Laboratory Shane Kennett Exponent Failure Analysis Associates Cheolmin Park Yonsei University 